\title{
Fundamental of Sequence System
}

\author{
Awadh International
}

ABSTRACT: It is a most advantage international level discovery for physics and technology. It solve many problems based on several field of life such as physical education, science, corporate world, engineering, industrial state, space science, mathematics, nuclear science, administration field, pre-planning, management and computer science etc. It is more easy and its representation is so more easy. We can easly provided this system in calculator, computer and many other technology based product.

Key Word : $\quad{ }_{P}, \sqrt{P},(.), R,.{\sqrt[s]{\left|P_{n}\right|}}^{n}$

\section{Introduction:}

The present is time of science and technology. Where physics and mathematics involve there produce technology. So it is a creation of technology. Right now we solve many problems by using plus, minus, multiplication, sin, cos, tan, cot, sec, cosec, calculus, statics, mechanism, integer, root, equation, matrix, determinant, log and many other formulae and theorem. It can be used to provent duplicasy. The sequence system is a fundamental theory to develop science, engineering and technology as well as mathematics.

\section{Description:}

\section{Concept Of Sequence System:-}

1.1 Consider a system, organised through any regular process and it's happen again and again with something other differences in any sequence, during the process, as follows

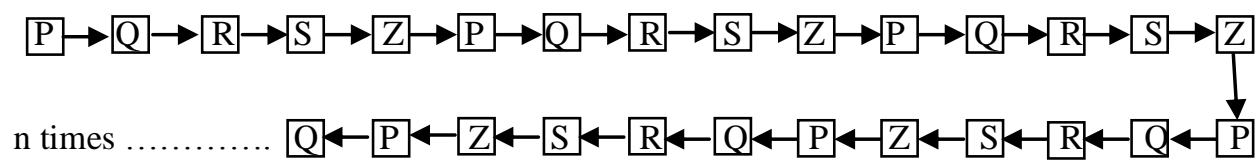

Then, it can be represented as

$$
{\sqrt[S]{\mathrm{A}_{(\mathrm{P}, \mathrm{S}, 5)}}}^{\mathrm{n}}
$$

where every $5^{\text {th }}$ step of this process is Z.

Here, consider every step as a process and $\mathrm{P}$ to $\mathrm{Z}$ as a group. The above is known as sequence system. The sequence system is a Managing Director, For status of any step \& many other information, we will be used remainder theory-

We know that if,

$\mathrm{X} \div \mathrm{Y}=\mathrm{Z}$

Where

$$
\begin{aligned}
& \text { then } \mathrm{X}=\mathrm{Y} \times \mathrm{Z} \\
& \mathrm{X}=\text { Dividend } \\
& \mathrm{Y}=\text { Divisor } \\
& \mathrm{Z}=\text { quotient }
\end{aligned}
$$

But if $\mathrm{X} \div \mathrm{Y} \neq \mathrm{Z}$ then

$$
\mathrm{X} \div \mathrm{Y}=\mathrm{Z} \text {.. } \mathrm{R} \text { where } \mathrm{Z} \text { is quotient and } \mathrm{R} \text { is remainder. }
$$

$\mathrm{X} \div \mathrm{Y}=\mathrm{Z} . \mathrm{R}$ will be known as remainder theorem.

And read as $-\mathrm{X}$ divided by $\mathrm{Y}$ or $\mathrm{X}$ upon $\mathrm{Y}$ is equal to $\mathrm{Z}$ remainder $\mathrm{R}$, and the symbol "(..)" denoted as remainder.

\section{Rules-}

For status of the process, we will be used remainder theory as following manners as the rules When the step, whose we required the status is divided by total number of step under a group as described sequence, then the process -

quotients contains no remainder, quotient $=$ group number quotient contain any remainder, 


$$
\text { quoitent }+1=\text { group number }
$$

\section{And for status of the step of process-}

If remainder is 1 , then the step will be $P$.

If remainder is 2 , then the step will be $\mathrm{Q}$.

If remainder is 3 , then the step will be $\mathrm{R}$.

If remainder is 4 , then the step will be $S$.

If remainder is 0 , then the step will be $Z$.

Now, consider $\mathrm{P}$ to $\mathrm{Z}$ as a group. To find out the status of $15^{\text {th }}$ and $19^{\text {th }}$ step

Given that, Total number of step under group $=5$, then by using remainder theory-

$$
15 \div 5=3 . .0
$$

We know that if quotient contains no remainder then,

Here remainder is zero so step will $\mathrm{Z}$.

$$
\text { group number }=\text { quotient }
$$

so, $\quad$ group number $=3$,

Hence the $15^{\text {th }}$ step will be under group number 3 and status of kinds of process will be $\mathrm{Z}$.

For status of step number 19 ,

$$
19 \div 5=3 . .4
$$

We know that if quotient contains any remainder then, group number $=$ quotient +1

$$
\text { so, group number }=3+1=4
$$

Hence the step number $19^{\text {th }}$ exist in group number 4 and type will be $S$.

\subsection{Fundamental-1}

\section{Fundamental of Sequence System}

To manufacture a product of 150 litre Z,50 litre solution (A)diluted with 60 litre solution (B)to produce solution 110 litre $(\mathrm{C})$, now it is heated upto $80^{\circ} \mathrm{C}$, the solution 70 litre $\mathrm{D}$ is prepared, now it is diluted with 30 litre solution $\mathrm{E}$, the 100 litre solution of $\mathrm{F}$ is occurred next it is freezited upto $-2^{\circ} \mathrm{C}$ with a solid $\mathrm{G}(50 \mathrm{kgs})$ to occure $\mathrm{H}$, it produce products I (150 ltre), now it is sealed and packed to sell in market. There some destroy material are occurred which collect in a container $(\mathrm{J})$. The process is completed and a product $\mathrm{Z}$ (150 ltre) will be ready to sport. The whole process completed in 11 steps its process can be represented on paper as a-

$$
\overbrace{\mathrm{P}_{(\mathrm{A}, \mathrm{J}, 11)}}^{\mathrm{s}}
$$

Where $\mathrm{P}$ shows process \& $\mathrm{s}$ shows that every $11^{\text {th }}$ step of this process is $\mathrm{Z}$.

This is a process of production for one unit $Z$. For more production it is done again and again as following-

$$
\mathrm{A} \rightarrow \mathrm{B} \rightarrow \mathrm{C} \rightarrow \mathrm{D} \rightarrow \mathrm{E} \rightarrow \mathrm{F} \rightarrow \mathrm{G} \rightarrow \mathrm{H} \rightarrow \mathrm{I} \rightarrow \mathrm{J} \rightarrow \mathrm{Z} \rightarrow \mathrm{A} \rightarrow \mathrm{B} \rightarrow \mathrm{C} \rightarrow \mathrm{D} \rightarrow \mathrm{E} \rightarrow \mathrm{F} \rightarrow \mathrm{G} \rightarrow \mathrm{H} \rightarrow \mathrm{I} \rightarrow \mathrm{J} \rightarrow \mathrm{Z} .
$$
...n times

\subsection{Fundamental-2}

If a system is work done in the form of

$\mathrm{P}_{1} \rightarrow \mathrm{P}_{2} \rightarrow \mathrm{P}_{3} \rightarrow \mathrm{P}_{4} \rightarrow \mathrm{P}_{5} \rightarrow \mathrm{P}_{6} \rightarrow \mathrm{P}_{7} \rightarrow \mathrm{P}_{8} \rightarrow \mathrm{P}_{9} \rightarrow \mathrm{P}_{10} \rightarrow \mathrm{P}_{11} \rightarrow \mathrm{P}_{12} \rightarrow \mathrm{P}_{13} \rightarrow \mathrm{P}_{14}$ ..n times<smiles>[In]=CC=[Tl]</smiles>

\subsection{Fundamental-3}

If a system is work done in the form of $\mathrm{P}_{1} \rightarrow \mathrm{P}_{2} \rightarrow \mathrm{P}_{3} \rightarrow \mathrm{P}_{4} \rightarrow \mathrm{X} \rightarrow \mathrm{P}_{6} \rightarrow \mathrm{P}_{7} \rightarrow \mathrm{P}_{8} \rightarrow \mathrm{P}_{9} \rightarrow \mathrm{X} \rightarrow \mathrm{P}_{11} \rightarrow \mathrm{P}_{12} \rightarrow \mathrm{P}_{13} \rightarrow \mathrm{P}_{14} \ldots \ldots \ldots \ldots$ times $=$ $\mathrm{S}$ shows that every $5^{\text {th }}$ step of this process is $\mathrm{X}$.<smiles>CC1CCCC1</smiles>
$\mathrm{n}=1$

\subsection{Fundamental-4}

$\mathrm{x}_{1}, \mathrm{x}_{2}, \mathrm{x}_{3}, \mathrm{P}, \mathrm{x}_{5}, \mathrm{x}_{6}, \mathrm{x}_{7}, \mathrm{P}, \mathrm{x}_{9}, \mathrm{x}_{10}, \mathrm{x}_{11}, \mathrm{P}, \mathrm{x}_{13}, \mathrm{x}_{14}, \mathrm{x}_{15}, \mathrm{P}, \mathrm{x}_{17}, \mathrm{x}_{18}, \mathrm{x}_{19}, \mathrm{P}, \mathrm{x}_{21}$ $\mathrm{n}$ times $=$

$\mathrm{S}$ shows that every $4^{\text {th }}$ term of this process is $\mathrm{P}$.

\subsection{Fundamental-5}

$2<4<6<8<10<12<14$ n times

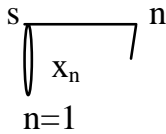

$=\bigodot_{x=1} 2 x^{n}$ 
2.6 Fundamental-6

n times

$.80>70>60>50>40>30>20>10$

2.7 Fundamental-7

$2-4+6-8+10-12+$ n times

\subsection{Fundamental -8}

$3.6+9.12+15.18+21.24+$

n times $=$

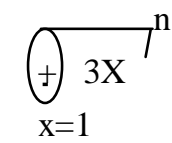

2.9 Fundamental-9:- Matrix/Determinate type

$\begin{array}{lllllllllll}\text { A } & \text { A } & \text { A } & \text { A } & \text { A } & \text { A } & \text { A } & \text { A } & \text { A } & \text { A } \\ \text { A } & \text { A } & \text { A } & \text { A } & \text { A } & \text { A } & \text { A } & \text { A } & \text { A } & \text { A } \\ \text { A } & \text { A } & \text { A } & \text { A } & \text { A } & \text { A } & \text { A } & \text { A } & \text { A } & \text { A }=\| \text { A }{ }_{10}{ }^{\text {n }} \\ \text { A } & \text { A } & \text { A } & \text { A } & \text { A } & \text { A } & \text { A } & \text { A } & \text { A } & \text { A } \\ . . & . . & . . & . . & . . & . . & . . & . . & . . & . . & \\ . . & . . & . . & . . & . . & . . & . . & . . & . . & . . & \end{array}$

\subsection{Fundamental-10}

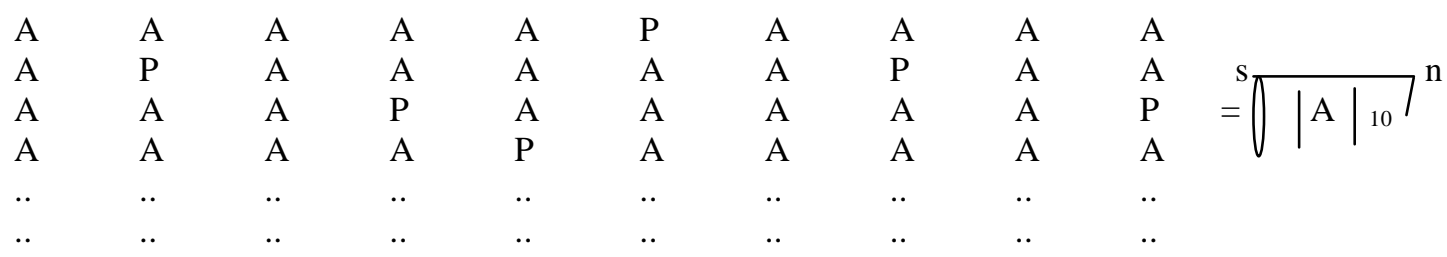

S shows that every $6^{\text {th }}$ term of this system is P.

\subsection{Fundamental-11}

$\begin{array}{lll}\mathrm{P}_{1} & \mathrm{P}_{6} & \mathrm{P}_{11} \\ \mathrm{P}_{2} & \mathrm{P}_{7} & \mathrm{P}_{12} \\ \mathrm{P}_{3} & \mathrm{P}_{8} & \mathrm{P}_{13} \\ \mathrm{P}_{4} & \mathrm{P}_{9} & \mathrm{P}_{14} \\ \mathrm{P}_{5} & \mathrm{P}_{10} & \mathrm{P}_{15}\end{array}$

\subsection{Fundamental -12}

If there is a system

$\begin{array}{llllllllll}\mathrm{P}_{1} & \mathrm{P}_{2} & \mathrm{P}_{3} & \mathrm{P}_{4} & \mathrm{P}_{5} & \mathrm{P}_{1} & \mathrm{P}_{2} & \mathrm{P}_{3} & \mathrm{P}_{4} & \mathrm{P}_{5} \\ \mathrm{P}_{1} & \mathrm{P}_{2} & \mathrm{P}_{3} & \mathrm{P}_{4} & \mathrm{P}_{5} & \mathrm{P}_{1} & \mathrm{P}_{2} & \mathrm{P}_{3} & \mathrm{P}_{4} & \mathrm{P}_{5} \\ \mathrm{P}_{1} & \mathrm{P}_{2} & \mathrm{P}_{3} & \mathrm{P}_{4} & \mathrm{P}_{5} & \mathrm{P}_{1} & \mathrm{P}_{2} & \mathrm{P}_{3} & \mathrm{P}_{4} & \mathrm{P}_{5} \\ \mathrm{P}_{1} & \mathrm{P}_{2} & \mathrm{P}_{3} & \mathrm{P}_{4} & \mathrm{P}_{5} & \mathrm{P}_{1} & \mathrm{P}_{2} & \mathrm{P}_{3} & \mathrm{P}_{4} & \mathrm{P}_{5}\end{array}$

Consider $\mathrm{P}_{1}, \mathrm{P}_{2}, \mathrm{P}_{3}, \mathrm{P}_{4}, \mathrm{P}_{5}$ as a group organized in 10 row.

Then under sequence system it can be written as.

$$
{\left.\sqrt{\mathrm{P} \mid}\right|_{(1,5,10)}}^{\mathrm{n}}
$$

For status of group and kind of member - 
Applying remainder theory,

If any term more than 5 divided by 5 , then

When quotients have no remainder then the quotient will be group number

When quotient have any remainder then the quotient +1 will be group number, and

If remainder is 1 the step will be $\mathrm{P}_{1}$

If remainder is 2 the step will be $\mathrm{P}_{2}$

If remainder is 3 the step will be $P_{3}$

If remainder is 4 the step will be $\mathrm{P}_{4}$

For row-

If remainder is 0 the step will be $\mathrm{P}_{5}$

If any term more than 10 divided by 10 then -

If quotients have no remainder the quotient will be column number.

If quotient have any remainder the (32uotient+1) will be column number.

\subsection{Fundamental -13}

If any system organized as -

$\begin{array}{llllllll}\mathrm{X}_{1} & \mathrm{X}_{2} & \mathrm{X}_{3} & \mathrm{X}_{4} & \mathrm{X}_{5} & \mathrm{X}_{6} & \mathrm{X}_{7} & \mathrm{X}_{8} \\ \mathrm{X}_{9} & \mathrm{X}_{10} & \mathrm{X}_{11} & \mathrm{X}_{12} & \mathrm{X}_{13} & \mathrm{X}_{14} & \mathrm{X}_{15} & \mathrm{X}_{16} \\ \mathrm{X}_{17} & \mathrm{X}_{18} & \mathrm{X}_{18} & \mathrm{X}_{19} & \mathrm{X}_{21} & \mathrm{X}_{22} & \mathrm{X}_{23} & \mathrm{X}_{24}\end{array}$

Then under sequence system it can be written as

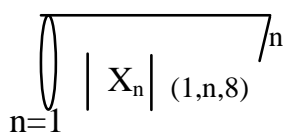

\section{For column}

Applying remainder theory,

When any term more than 8 divided by 8 then -

If quotients have no remainder the quotient will be column number.

If quotient have any remainder the (quoitent +1 ) will be column number.

\subsection{Fundamental -14}

If any system organized as -

$\begin{array}{llllllll}\mathrm{X}_{1} & \mathrm{X}_{4} & \mathrm{X}_{7} & \mathrm{X}_{10} & \mathrm{X}_{13} & \mathrm{X}_{16} & \mathrm{X}_{19} & \mathrm{X}_{22} \\ \mathrm{X}_{2} & \mathrm{X}_{5} & \mathrm{X}_{8} & \mathrm{X}_{11} & \mathrm{X}_{14} & \mathrm{X}_{17} & \mathrm{X}_{20} & \mathrm{X}_{23} \\ \mathrm{X}_{3} & \mathrm{X}_{6} & \mathrm{X}_{9} & \mathrm{X}_{12} & \mathrm{X}_{15} & \mathrm{X}_{18} & \mathrm{X}_{21} & \mathrm{X}_{24}\end{array}$

Then under sequence system it can be written as

For row

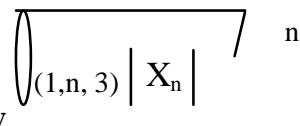

Applying remainder theory,

When any term more than 3 divided by 3 then -

If quotients have no remainder the quotient will be row number.

If quotient have any remainder the (quoitent+1) will be row number.

\subsection{Physics}

\section{Application}

3.1.1 Radio activity :The process by which a nucleus of an unstable atom loses energy by emitting particles of ionizing radiation. A material that spontaneously emits this kind of radiation which include the emission of energetic $\alpha$-particle, $\beta$-particle and $\gamma$-particle, consider as radio activity.

$\alpha, \beta, \gamma$ decay mean loses of energies. When -

One $a$ particle decay -

loss of atomic number 2.

loss of atomic mass number 4 .

One $\beta$, particle decay-

increase atomic number 1

increase atomic mass number zero.

If $\mathrm{Z}$ is a atom contain atomic mass number 210 and atomic number 90 . 
then under sequence system it can be generally shows as -

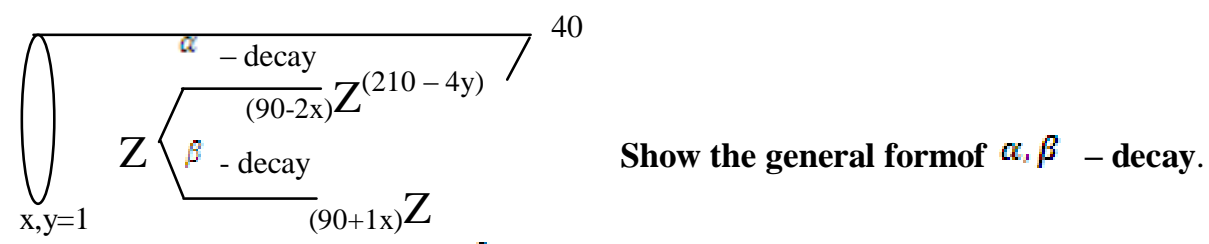

If we have find out the status after $8 \alpha, \beta$ decay, then

It is given that,

$$
\begin{aligned}
\mathrm{X}, \mathrm{y} & =8 \\
(90-2 \mathrm{x}) & \mathrm{Z}^{210-4 y}={ }_{(90-16)} \mathrm{Z}^{210-32}={ }_{74} \mathrm{Z}^{178}
\end{aligned}
$$

After $8 \propto, \beta$-decay the atom will be contains atomic number 74 and atomic mass number 178.

\subsection{Industrial state -}

Consider A is product of $100 \mathrm{gm}$. per piece. To manufactured it's $45 \mathrm{gmX}, 20 \mathrm{gmY}$ and $35 \mathrm{gmZ}$ material are used. Find out the total material used for 200 piece. This whole process can be written as -

$$
\bigcup_{(\mathrm{x}, \mathrm{y}, \mathrm{Z}=1)}(45 \mathrm{X}+20 \mathrm{Y}+35 \mathrm{Z}) 7^{\mathrm{n}}
$$

Here $45 \mathrm{X}+20 \mathrm{Y}+35 \mathrm{Z}$ is not an equation

For 200 piece-

$$
\mathrm{X}, \mathrm{Y}, \mathrm{Z}=200
$$

So, $\quad 45 \mathrm{X}+20 \mathrm{Y}+35 \mathrm{Z}$ is,

$$
\begin{aligned}
& X=45 \times 200=9000 \\
& Y=20 \times 200=4000 \\
& Z=35 \times 200=7000
\end{aligned}
$$

Total material used for 200 piece $=45 \times 200+20 \times 200+35 \times 200=20000 \mathrm{gm}=20 \mathrm{Kg}$

Hence $20 \mathrm{Kg}$ material used for 200 piece

\subsection{In corporate world (To provant duplicasy)}

A manufacturing company launches your product in market, To prevent duplicity we used coding system as

\begin{tabular}{|l|c|c|c|c|c|c|c|c|c|c|c|c|c|c|c|c|}
\hline Batch No. & \multicolumn{10}{|c|}{01} \\
\hline Lot No. & 01 & 02 & 03 & 04 & 05 & 06 & 07 & 08 & 09 & 10 & 11 & 12 & 13 & 14 & 15 & 16 \\
\hline $\begin{array}{l}\text { Product } \\
\text { Code }\end{array}$ & D & A & B & P & Q & C & R & S & D & A & B & P & Q & C & R & S \\
\hline
\end{tabular}

\begin{tabular}{|l|c|c|c|c|c|c|c|c|c|c|c|c|c|c|c|c|}
\hline $\begin{array}{l}\text { Batch } \\
\text { No. }\end{array}$ & \multicolumn{11}{|c|}{02} \\
\hline Lot No. & 01 & 02 & 03 & 04 & 05 & 06 & 07 & 08 & 09 & 10 & 11 & 12 & 13 & 14 & 15 & 16 \\
\hline $\begin{array}{l}\text { Product } \\
\text { Code }\end{array}$ & D & A & B & P & Q & C & R & S & D & A & B & P & Q & C & R & S \\
\hline
\end{tabular}

\begin{tabular}{|l|c|c|c|c|c|c|c|c|c|c|c|c|c|c|c|c|}
\hline $\begin{array}{l}\text { Batch } \\
\text { No. }\end{array}$ & \multicolumn{10}{|c|}{03} \\
\hline Lot No. & 01 & 02 & 03 & 04 & 05 & 06 & 07 & 08 & 09 & 10 & 11 & 12 & 13 & 14 & 15 & 16 \\
\hline $\begin{array}{l}\text { Product } \\
\text { Code }\end{array}$ & D & A & B & P & Q & C & R & S & D & A & B & P & Q & C & R & S \\
\hline
\end{tabular}

Find out the product code and batch number of lot number 453 and 640 .

$\mathrm{Sol}^{\mathrm{n}}$ :- $\quad$ Under sequence system with use of remainder theory, we write it.

$$
\mathrm{X}_{(1,16,16)}^{\mathrm{n}}
$$

Where $\mathrm{X}$ is a process organized such that $\mathrm{X}=\mathrm{D} \rightarrow \mathrm{A} \rightarrow \mathrm{B} \rightarrow \mathrm{P} \rightarrow \mathrm{Q} \rightarrow \mathrm{C} \rightarrow \mathrm{R} \rightarrow \mathrm{S} \rightarrow \mathrm{D} \rightarrow \mathrm{A} \rightarrow \mathrm{B} \rightarrow \mathrm{P} \rightarrow \mathrm{Q} \rightarrow \mathrm{C} \rightarrow \mathrm{R} \rightarrow \mathrm{S}$ in sequence consider as a group. 
For the Batch No. and lot code no. 453

$453 \div 16=28 . .5$, so the lot number 453 is product of batch no 28 and lot code is $\mathrm{Q}$.

\subsection{Engineering \& Technology}

3.4.1 Example - The railway department prepare of a project to make up $100 \mathrm{~km}$ electric root with the help of 10,000 pole such as every five pole is master pole and all other pole is as general pole. Find out group and type of $6372^{\text {th }}, 7575^{\text {th }}, 8387^{\text {th }}$ pole, considering 1 to 5 pole as a group.

$\mathrm{Sol}^{\mathrm{n}}$ - $\quad$ Under sequence system with use of remainder theory, it can be written as -

$$
\begin{aligned}
& \mathrm{A}, \mathrm{A}, \mathrm{A}, \mathrm{A}, \mathrm{P}, \mathrm{A}, \mathrm{A}, \mathrm{A}, \mathrm{A}, \mathrm{Z}, \ldots \ldots . .1000=\prod_{\mathrm{S}}^{\mathrm{X} \mathrm{X}_{(\mathrm{A}, \mathrm{A}, 5}}{ }^{1000} \mathrm{X}=\mathrm{A}, \mathrm{A}, \mathrm{A}, \mathrm{A}, \mathrm{Z} \text { in sequence, } \mathrm{S} \text { show every five poles as a master pole. }
\end{aligned}
$$

There are total number of member under group $=5$

Status of $6372^{\text {th }}$ pole -

$6372 \div 5=1274 . .2$

When quotient have any remainder group $=$ quotient +1

$$
\begin{aligned}
\text { group } & =1274+1 \\
& =1275
\end{aligned}
$$

Hence the $6372^{\text {th }}$ pole will be under group 1275 and $2^{\text {nd }}$ pole $\left(2^{\text {nd }}\right.$ pole is A)

For status of $7575^{\text {th }}$ pole,

$7575 \div 5=1515 . .0$

When quotient have no remainder.

The group $=$ quotient

hence $7575^{\text {th }}$ pole is the member of group $1515^{\text {th }}$ and it is master pole $\mathrm{Z}$.

For status of $8387^{\text {th }}$ term

$8387 \div 5=1235 . .2$

When quotient contains any remainder then(qoitent+1) is the group number, so

Group number $\quad=1235+1$

$=1236$

Hence, $8387^{\text {th }}$ pole is member of 1236 it will be $2^{\text {nd }}$ pole.

\subsubsection{Engineering \& Technology}

If there is a system contains many step of process such as $\mathrm{x}_{1}, \mathrm{x}_{2}, \mathrm{x}_{3}, \mathrm{x}_{4}, \mathrm{x}_{5}, \mathrm{x}_{6}, \mathrm{x}_{7}, \mathrm{x}_{8}, \mathrm{x}_{9}, \mathrm{x}_{10}, \mathrm{P}, \mathrm{x}_{1}, \mathrm{x}_{2}, \mathrm{x}_{3}, \mathrm{x}_{4}$, $\mathrm{x}_{5}, \mathrm{x}_{6}, \mathrm{x}_{7}, \mathrm{x}_{8}, \mathrm{x}_{9}, \mathrm{x}_{10}, \mathrm{P}, \mathrm{x}_{1}, \mathrm{x}_{2}, \mathrm{x}_{3}, \mathrm{x}_{4}, \mathrm{x}_{5}, \mathrm{x}_{6}, \mathrm{x}_{7}, \mathrm{x}_{8}, \mathrm{x}_{9}, \mathrm{x}_{10}, \mathrm{P} \mathrm{x}_{1}, \mathrm{x}_{2}, \mathrm{x}_{3}, \mathrm{x}_{4}, \mathrm{x}_{5}, \mathrm{x}_{6}, \mathrm{x}_{7}, \mathrm{x}_{8}, \mathrm{x}_{9}, \ldots . \mathrm{n}$

Whose every $11^{\text {th }}$ step is $\mathrm{P}$ as a head then this system can be represented as -

$$
\overbrace{\mathrm{x}_{(1,10,11)}}^{\mathrm{s}}
$$

where $\mathrm{x}$ is a system such that $\mathrm{x}=\mathrm{x}_{1}, \mathrm{x}_{2}, \mathrm{x}_{3}, \mathrm{x}_{4}, \mathrm{x}_{5}, \mathrm{x}_{6}, \mathrm{x}_{7}, \mathrm{x}_{8}, \mathrm{x}_{9}, \mathrm{x}_{10}, \mathrm{Z}$ consider as a group. Now find out the status of step of process $25^{\text {th }}$ and $35^{\text {th }}$.

Solution for status of member number 25 ,

$$
25 \div 11=2 . .3
$$

We know that if quotient contains any remainder,

$$
\begin{aligned}
& \text { group number }=\text { quotient }+1 \\
& \text { group number }=2+1 \\
& \text { group number }=3
\end{aligned}
$$

Here remainder 3. So, group will be $3 \&$ Type of process is $x_{3}$.

Hence $25^{\text {th }}$ member exist group number 3 and member will $x 3$

For Status of member number $33^{\text {th }}$

$$
33 \div 11=3 . .0
$$

We know that if quotient contains no remainder

$$
\begin{aligned}
& \text { Group number }=\text { quotient } \\
& \text { Groupnumber }=3
\end{aligned}
$$

Here remainder zero. So, group will be 3 \& Type of process is Z.

\subsubsection{Engineering \& Technology}

Consider a series - 2, 4, 6, 8, - 10, 12, 14, 16, 18, - 20, figure Now find out its $432^{\text {th }}$ and $620^{\text {th }}$ term.

$\mathrm{Sol}^{\mathrm{n}}$ : 
$\prod_{\mathrm{X}=1}^{\mathrm{S}} 2 \mathrm{X} 7^{\mathrm{n}} \quad$ Where $\mathrm{s}$ shows that every $5^{\text {th }}$ term of this system is as minus term.

For $432^{\text {th }}$ term using remainder concept.

$432 \div 5=86 . .2$ there is remainder two. So, it is not minus figure.

Therefore, the position of $432^{\text {th }}$ term will be.

given $\mathrm{X}=432$

So, $2 \mathrm{X}=432 \mathrm{X} 2=864$

hence it $432^{\text {th }}$ terms will be 864 .

For $620^{\text {th }}$ term

$620 \div 5=124 . .0$

here remainder is zero hence it is under minus figure.

given that $\mathrm{X}=620$

So. $2 \mathrm{X}=620 \mathrm{X} 2=1240$

hence the $620^{\text {th }}$ term will be $(-1240)$.

\subsection{Distance, speed and time:-}

A Train have contained speed $80 \mathrm{~km} / \mathrm{hour}$. Find out the distance after 8 hour?

$\mathrm{Sol}^{\mathrm{n}}$ : Under sequence system it can be written as -

$$
\sqrt{80 X}^{n}
$$

$\mathrm{X}=1$

given that,

$$
\mathrm{X}=8 \text { then, }
$$

$80 \times 8=640 \mathrm{~km}$

The train have gone $640 \mathrm{Km}$ after 8 hour.

\subsection{Administration field}

A university declares your annual examination. The seating plan make up as - there are 16 rooms, 50 students in every room, 10 students in every column, total student are 768 as given below -

\begin{tabular}{|c|c|c|c|c|c|}
\hline 1 & $\mathrm{X}_{1}$ & $\mathrm{X}_{1}$ & $\mathrm{X}_{1}$ & $\mathrm{X}_{1}$ & $\mathrm{X}_{1}$ \\
\hline 2 & $\mathrm{Y}_{3}$ & $\mathrm{Y}_{3}$ & $\mathrm{Y}_{3}$ & $\mathrm{Y}_{3}$ & $\mathrm{Y}_{3}$ \\
\hline 3 & $\mathrm{X}_{2}$ & $\mathrm{X}_{2}$ & $\mathrm{X}_{2}$ & $\mathrm{X}_{2}$ & $\mathrm{X}_{2}$ \\
\hline 4 & $\mathrm{Y}_{1}$ & $\mathrm{Y}_{1}$ & $\mathrm{Y}_{1}$ & $\mathrm{Y}_{1}$ & $\mathrm{Y}_{1}$ \\
\hline 5 & $\mathrm{X}_{3}$ & $\mathrm{X}_{3}$ & $\mathrm{X}_{3}$ & $\mathrm{X}_{3}$ & $\mathrm{X}_{3}$ \\
\hline 6 & $\mathrm{Y}_{2}$ & $\mathrm{Y}_{2}$ & $\mathrm{Y}_{2}$ & $\mathrm{Y}_{2}$ & $\mathrm{Y}_{2}$ \\
\hline 7 & $\mathrm{Y}_{4}$ & $\mathrm{Y}_{4}$ & $\mathrm{Y}_{4}$ & $\mathrm{Y}_{4}$ & $\mathrm{Y}_{4}$ \\
\hline 8 & $\mathrm{X}_{5}$ & $\mathrm{X}_{5}$ & $\mathrm{X}_{5}$ & $\mathrm{X}_{5}$ & $\mathrm{X}_{5}$ \\
\hline 9 & $\mathrm{Y}_{5}$ & $\mathrm{Y}_{5}$ & $\mathrm{Y}_{5}$ & $\mathrm{Y}_{5}$ & $\mathrm{Y}_{5}$ \\
\hline 10 & $\mathrm{X}_{4}$ & $\mathrm{X}_{4}$ & $\mathrm{X}_{4}$ & $\mathrm{X}_{4}$ & $\mathrm{X}_{4}$ \\
\hline
\end{tabular}

above is status of one room. Find out the status of $437^{\text {th }}$ and $670^{\text {th }}$ student. Here,

1- $\quad \mathrm{X}_{1}$ mean student of B.Sc. I

2- $\quad \mathrm{X}_{2}$ mean student of B.Sc. II

3- $\quad \mathrm{X}_{3}$ mean student of B.Sc. III

4- $\quad \mathrm{X}_{4}$ mean student of M.Sc. I

5- $\quad X_{5}$ mean student of M.Sc. II

6- $\quad Y_{1}$ mean student of B.A. I

7- $\quad \mathrm{Y}_{2}$ mean student of B.A. II

8- $\quad \mathrm{Y}_{3}$ mean student of B.A. III

9- $\quad Y_{4}$ mean student of M.A. I

10- $\quad Y_{5}$ mean student of M.A. II

$\mathrm{Sol}^{\mathrm{n}}$ : $\quad$ Under sequence system it can be written as -

$$
\widehat{(1,10,5)}|\mathrm{Z}|^{768}
$$


$Z: Z=X_{1}, Y_{3}, X_{2}, Y_{1}, X_{3}, Y_{2}, Y_{4}, X_{5}, X_{4}$ in sequence under first row.

For status of room, for $\mathbf{4 3 7}^{\text {th }}$ student.

given that,

Total number of student in room $=50$

hence $437 \div 50=8 . .37$

So, $\quad$ qoitent $+1=8+1$

$$
=9
$$

therefore student seated in room number 9 .

For column status -

There are 10 student in a column, hence.

$$
37 \div 10=3 . .7
$$

So,the student seated in column number

$$
\begin{aligned}
\text { qoitent }+1 & =3+1 \\
& =4
\end{aligned}
$$

Hence the $437^{\text {th }}$ student seated in room number 9 , seat number $7^{\text {th }}$ of column 4 .

For status of student number 670 ,

$$
\begin{aligned}
& 670 \div 50=13 . .20 \\
& 20 \div 10=2 . .00
\end{aligned}
$$

Hence the student status is, room number 14 , column number 2, last student.

\subsection{Economics}

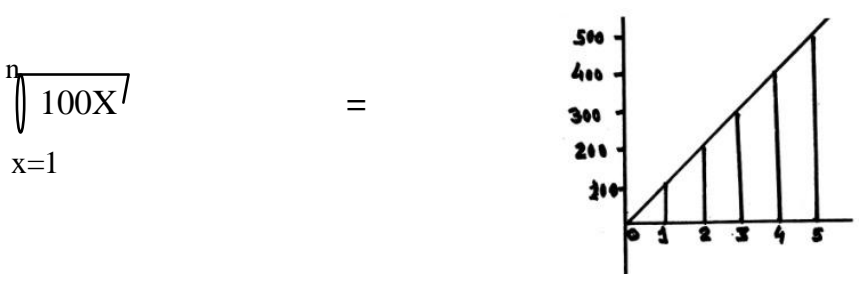

\section{Some other mathematical fundamental \& Example-}

4.1 If there is a series such as $3,9,27,81$, $\mathrm{n}$ then under sequence system it can be written as -

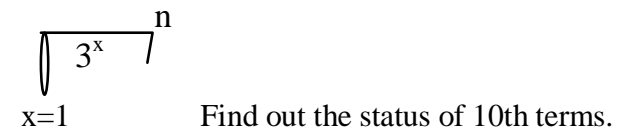

Solution-

$$
\begin{aligned}
& \text { given that } \\
& x=10 \\
& \text { hence } \\
& 3^{10}=3 \times 3 \times 3 \times 3 \times 3 \times 3 \times 3 \times 3 \times 3 \times 3 \\
& 3^{10}=59049
\end{aligned}
$$

Hence the $10^{\text {th }}$ term of this series is 59049 .

4.2 A series $2+4+6+8+10+12-14+16+18+20+22 \ldots \ldots \ldots$ whose $7^{\text {th }}, 80^{\text {th }}, 108^{\text {th }}$ terms under minus figure,it can be written as -

$$
\prod_{x=1}^{s}{ }^{n}
$$

$\mathrm{X}: \mathrm{X}=2+4+6+8$

Find out the $67^{\text {th }} \& 80^{\text {th }}$ term of this series

\section{Solution-}

For $67^{\text {th }}$ term

$1^{\text {st }}$ we check that it is not under $7^{\text {th }}, 80^{\text {th }}, \& 108^{\text {th }}$ term.

Given that

$\mathrm{X}=67$

Then $2 . X=2 X 67=134$ 
Hence the $67^{\text {th }}$ term of this series is 134

for $80^{\text {th }}$ term.

$1^{\text {st }}$ we check that it is under $80^{\text {th }}$ term which is under minus figure

Here given that

$\mathrm{X}=80$ then

2.X $=(-) 2$ X $80=(-) 160$

Hence its $80^{\text {th }}$ will be $(-160)$

4.3 Consider a series $3-5+7-9+11-13+\ldots$ n times, find out its $435^{\text {th }}$ and $640^{\text {th }}$ term.

Solution-

Under sequence system it can be written as

$3-5+7-9+11-13+\ldots \ldots \ldots \ldots . n$ times $\quad=\quad \overbrace{\mathrm{X}=1}^{+}$

\section{For status of $435^{\text {th }}$ term.}

From remainder theorem.

There are two type of member involve is this system

hence

$435 \div 2=217 . .1$

Here remainder is one hence it is under (+) Sign,

So, Given that

$\begin{aligned} X & =435 \\ \text { Then, } \quad(2 \times 435+1) & =870+1=871\end{aligned}$

hence its $435^{\text {th }}$ term will be 871 .

For status of $640^{\text {th }}$ term

$640 \div 2=320 . .0$

Given that

Here remainder is zero, so it is under (-) sign,

\section{Hence the $640^{\text {th }}$ term will be (-) 1281 .}

$$
\begin{gathered}
X=640, \quad \text { So } \\
(-)\{2 . X+1\}=(-)\{2 \times 640+1\}=(-) 1281 .
\end{gathered}
$$

\section{Conclusion :}

This is a new topic for physical science and technology which can be most useful at the time of preparation of any planning, project in the field of engineering and technology. The Fundamental of sequence system is most important.Itis advanatage of several field of life such as science and engineering, to issue currency, to provent duplicasy, to issue mobile recharges, public administration, corporate word, industrial state, mathematics, nuclear physics, space sciences etc. It is more easy to denote and understand.The symbol of remainder "(..)" how so easy to denote $\&$ it can be so easly to provide many system.

\section{Reference:}

[1]. Davenport, Harold (1999). The higher arithmetic: an introduction to the theory of numbers. Cambridge, UK: Cambridge University Press. p. 25ISBN 0-521-63446-6

[2]. Schwartzman, Steven (1994). "remainder (noun)". The words of mathematics : an etymological dictionary of mathematical terms used in english. Washington: Mathematical Association of AmericaISBN 9780883855119.

[3]. Fosnot and Dolk 2001. Young Mathematicians at Work: Constructing Multiplication and Division. Portsmouth, NH: Heinemann.

[4]. Walker, Jearl (2011). Principles of physics (9th ed.). Hoboken, N.J. : WileyISBN 0-470-56158-0.

[5]. Suchocki, John. Conceptual Chemistry, 2007. Page 119.

[6]. Radio-activity by Ernest Rutherford Phd. Encyclopedia Britannica Eleventh Edition. 